\title{
Escola experimental de primatas
}

\author{
Olavo de Faria Galvão \\ Romariz da Silva Barros \\ Aline Cardoso Rocha \\ Mariana Barreira Mendonça \\ Paulo Roney Kilpp Goulart
}

Universidade Federal do Pará

\begin{abstract}
Resumo
Aproximando, metaforicamente, os termos "laboratório" e "escola", o presente trabalho apresenta um programa de pesquisa experimental que visa desenvolver procedimentos para a construção de repertório simbólico em macacos-prego (Cebus apella). A metáfora "laboratório-escola" justifica-se pela semelhança dos procedimentos de treino de discriminações ou relações com programas de ensino de tarefas gradualmente mais complexas. O desafio tem sido o de manter o comportamento dos "aprendizes" sob controle das propriedades da tarefa programadas pelo experimentador. Os procedimentos são permanentemente avaliados em função da efetividade para produzir a planejada formação de relações não diretamente treinadas entre estímulos. Os resultados obtidos demonstram a aquisição do conceito de identidade e indicam a possibilidade de ensino de repertórios mais complexos, que são pré-requisitos para o comportamento simbólico e o possível aproveitamento de tecnologia para ensino desse tipo de repertório a pessoas com dificuldades no desenvolvimento de funcionamento simbólico convencional.
\end{abstract}

Palavras-chave: Escola para primatas, Comportamento simbólico, Equivalência de estímulos, Controle de estímulos, C. apella.

\begin{abstract}
Experimental school for primates.. Equating, metaphorically, the terms "lab" and "school", this article presents an experimental research program aiming to develop procedures to build symbolic repertories in capuchin monkeys (Cebus apella). The metaphor "lab-school" arises from the similarity of the training procedures, used to teach discriminations or stimulus relations, to teaching programs used to introduce gradually more complex tasks. The challenge has been to keep the behavior of the "learners" under the control of the task properties, as programmed by the experimenter. Results document the acquisition of generalized identity and indicate the possibility of teaching more complex repertoires that are prerequisite for symbolic behavior and the possible application of the developed technology in teaching such repertoires to persons with difficulties to develop conventional symbolic functioning.
\end{abstract}

Key words: Primates school, Symbolic behavior, Stimulus equivalence, Stimulus control, C. apella.

$\mathrm{O}$ presente trabalho descreve um programa de pesquisa nomeado "Escola Experimental de Primatas", sobre comportamento complexo em primatas não-humanos, e discute a validade dos estudos do comportamento de primatas para o conhecimento do comportamento humano.

Os conceitos interdependentes de adaptação e de habitat na teoria da seleção natural são básicos para a ciência do comportamento. A adaptação envolve a capacidade de viver e reproduzir-se dentro dos limites de variação das condições ambientais. A evolução da vida depende (1) de organismos que consigam interagir com seu ambiente imediato, do qual tiram seu sustento e no qual expelem os restos de seu metabolismo (2) de organismos que em larga medida têm características similares às de seus pais, mas que apresentam também variações em relação a eles. As variações que favorecem a capacidade de interagir com o ambiente imediato tendem a favorecer, também, a sobrevivência e a reprodução dos indivíduos que ostentam aquela característica, em um dado conjunto de condições ambientais.

O contato dos indivíduos com o seu ambiente se dá através do comportamento, cuja infraestrutura, isto é, o conjunto dos receptores sensíveis a mudanças ambientais de luz, ca- 
lor, pressão, composição química, e o dos efetores que realizam os movimentos, é herdada, de forma que as capacidades comportamentais lhes permitem interagir com seu ambiente de forma semelhante à de seus ancestrais. Das capacidades comportamentais herdadas, uma, estreitamente relacionada com a variabilidade comportamental necessária para a adaptação a um ambiente em constante mudança, é a capacidade de aprender, que se comprovou estar presente mesmo em organismos unicelulares (Jennings, 1906/1976; Peeke \& Herz, 1973). Para mais detalhes sobre a relação entre herança e ontogênese no comportamento complexo ver Bussab (2000) e Bussab e Ribeiro (1998), e sobre os limites das capacidades de aprendizagem de macacos-prego ver Visalberghi e Tomasello (1998).

A ciência do comportamento se preocupa em entender como os indivíduos e os grupos de indivíduos interagem com seu ambiente imediato para conseguir alimento, água, ar, condições adequadas de temperatura, pressão e umidade; como interagem com os indivíduos de sua espécie nas diversas fases da vida e com os indivíduos das inúmeras espécies que convivem no mesmo lugar. A nossa preocupação aqui se dirige para os processos comportamentais que permitem a interação dos indivíduos com o seu ambiente, particularmente no desenvolvimento de repertórios complexos, envolvendo relações arbitrárias entre estímulos, características do comportamento simbólico.

Do ponto de vista da análise evolutiva, podemos considerar a complexidade estrutural e funcional dos organismos na natureza como resultante da interação de um conjunto de processos simples, atuando em e sobre organismos gradualmente mais complexos (Donahoe \& Palmer, 1994). A história do comportamento complexo dos seres humanos se inicia muito antes da própria espécie humana existir na forma como hoje a conhecemos.

Comparando a cognição humana e animal na solução de problemas, Visalberghi e Limongelli (1994) demonstraram que um macaco-prego que havia resolvido um problema de tirar um doce de um tubo com uma vara, empurrando o doce para o lado sem armadilha - um furo no meio do tubo - continuava usando a estratégia de empurrar o doce enfiando a vara pelo lado mais distante do doce, mesmo com um tubo sem armadilha, indicando falta de compreensão do problema, mesmo após tê-lo resolvido.

Visalberghi e Tomasello (1998) analisaram esse e outros estudos de solução de problemas com primatas e com crianças e concluíram que crianças com mais de três anos compreendem e aplicam análise de causa e efeito para resolver problemas, enquanto os primatas e crianças de menos de três anos mostram aprendizagem do tipo "associativo", dependendo de muitas repetições do problema para resolvê-lo. Problema semelhante vem sendo descrito nos estudos de escolha segundo o modelo por identidade com animais, cujo desempenho vincula-se à estrita situação e estímulos ensinados (Lionello \& Urcuioli, 1998).

Em resumo, a noção de continuidade evolutiva entre as espécies e a concepção de que a complexidade resulta da interação de processos mais simples justificam a pesquisa sobre processos comportamentais em organismos não-humanos.

\section{Análise da aquisição de comportamentos complexos}

Em condições normais, uma criança aprende a falar as primeiras palavras ainda no primeiro ano de vida e seu repertório receptivo e ativo, isto é de compreensão e expressão, passa por um desenvolvimento tão fantástico quanto rápido. O seu repertório torna-se cada vez mais abrangente e adaptado a circunstâncias mais precisas do ambiente, sendo difícil a identificação dos processos interativos específicos em andamento.

Quando nos defrontamos com atrasos no desenvolvimento e precisamos criar condições especiais para uma criança adquirir repertórios que a maioria das crianças adquire sem precisar de atenção especial, vemo-nos diante da necessidade de entender o desenvolvimento normal para poder planejar essas condições. No sistema escolar, por exemplo, em que uma parcela significativa das crianças não alcança as metas de repertório acadêmico esperado, precisamos de instrumentos de análise que, em uma analogia com o microscópio usado na biologia, permitam a ampliação do objeto de estudo e possibilitem a visão, em detalhe, dos processos que levam ao sucesso e ao fracasso.

A análise do comportamento se propõe a medir passo a passo os processos comportamentais, e tornar visíveis as relações ambiente/comportamento que estão presentes na manutenção e modificação do repertório dos indivíduos (Catania, 1999; Sidman, 1995; Skinner, 1991). Ao estudar os processos comportamentais na interação imediata entre o indivíduo e seu ambiente, identificando funções de estímulos em situações de laboratório, a análise do comportamento complementa as abordagens culturalistas (Chaves, 2000), cujas construções teóricas envolvem o estudo interdisciplinar da complexidade comportamental, considerados os níveis de análise das diferentes ciências humanas.

O desenvolvimento de habilidades cognitivas nas pessoas normais é rápido, e possivelmente atingido por diferentes caminhos, o que torna o laboratório importante para a sua análise pois nele se pode restringir o número de eventos concomitantes, reproduzir conjuntos de condições e aumentar a possibilidade de reproduzir eventos comportamentais, e ter domínio sobre as alterações introduzidas no sistema. No laboratório se estabelecem relações funcionais entre ambiente e comportamento, depois usadas para interpretar o comportamento em situações complexas, nas quais provavelmente tais relações estão envolvidas.

Apesar do comportamento complexo, em condições normais, resultar da atuação de um número muito grande de variáveis, não se deve pensar a complexidade como resultado de processos caóticos e muito menos inacessíveis ao estudo e interpretação científica. Do mesmo modo, não se está autorizado a interpretar os fenômenos comportamentais complexos como resultado puro e simples das funções comportamentais verificadas no laboratório (Codo \& VasquesMenezes, 1999). 
Evidentemente, o estudo das condições necessárias para modificar o repertório de um indivíduo não se esgota no laboratório. $\mathrm{O}$ estudo de condições necessárias para produzir determinados repertórios pode ser, por exemplo, uma simulação de uma situação normal, dotada de condições de observação e registro. Assim, muitos relatos de pesquisa do comportamento não são estudos de laboratório, não são experimentos, mas descrevem observações em situações de ensino planejadas, com controle das condições ambientais.

$\mathrm{O}$ estudo das condições de ensino para pessoas com deficiência mental profunda, com linguagem pouco desenvolvida, tem esclarecido processos que, em pessoas normais, ocorrem tão rapidamente que se torna difícil detectar (Serna, Dube \& McIlvane, 1997).

Para ensinar uma criança ou adolescente com atraso no desenvolvimento de repertórios que crianças normais aprendem sem ensino explícito ou formal (como olhar nos olhos do interlocutor no momento de uma interação verbal ou repertórios escolares como ler uma palavra escrita), o termo análise se enche de sentido na medida em que cada mínimo progresso tem que ser observado e recompensado; cada novo repertório tem que ser praticado em diferentes contextos. Fica evidente o caráter hierárquico dos desempenhos e a necessidade de aprendizagem, passo a passo, de repertórios que, na criança normal, parecem surgir como mudanças qualitativas do tipo tudo ou nada (ver por exemplo, Dube, 1996).

\section{Por que uma escola experimental de primatas?}

Uma ciência do comportamento pode nos ajudar a entender a complexidade comportamental, estudando como as relações ambiente/comportamento se desenvolveram ao longo da história das espécies e como se desenvolvem ao longo da história dos indivíduos. Do ponto de vista da ciência, há uma continuidade na natureza, de forma que entender processos comportamentais em organismos mais simples contribui para a identificação e compreensão de processos comportamentais em organismos mais complexos como o homem.

A pesquisa com animais permitiu a demonstração experimental de processos comportamentais e a mensuração de relações funcionais, que se tornaram elementos fundamentais para a construção de instrumentos de análise e atuação em situações complexas como em escolas, no atendimento clínico ou em outras situações.

A pesquisa experimental com humanos tem, também, limitações que justificam o uso de animais como modelos simplificados (McIlvane \& Cataldo, 1996). É de particular importância a pesquisa com sujeitos não-humanos para as áreas de aplicação da Psicologia, com destaque para o ensino a pessoas com desenvolvimento severamente retardado.

\section{Um modelo animal para o comportamento simbólico?}

De acordo com Bates (1979), o comportamento simbólico é a compreensão ou uso, dentro ou fora de situações de comunicação, de uma relação entre um símbolo e seu referente, de tal maneira que o símbolo é tratado como pertencente a e/ ou substituível por seu referente em uma variedade de con- textos. Ao mesmo tempo, o símbolo é separável de seu referente, isto é, eles não se tornam a mesma coisa.

Apesar da dificuldade de definir comportamento simbólico de maneira compatível com as diversas áreas de conhecimento que o abordam, a definição apresentada pela lingüista Bates (1979) parece manter importantes pontos de contato com uma ainda iniciante abordagem analítico-comportamental do assunto. A definição de Bates trata o comportamento simbólico como comportamento controlado por relações arbitrárias entre estímulos (símbolos e seus referentes), de maneira que se tornem substituíveis um pelo outro em determinados contextos.

O modelo descritivo de classes de equivalência, surgido na análise do comportamento com inspiração em um modelo matemático de equivalência, consiste na verificação da possibilidade de substituição de estímulos arbitrariamente relacionados num determinado contexto em que estas relações foram construídas (Sidman, 1994; Sidman, 2000; Sidman \& Tailby, 1982). É patente a similaridade entre as definições. Formamos classes quando relacionamos nomes a coisas ou eventos ou quando relacionamos os sons da fala com a escrita correspondente. A base para o comportamento lingüístico e simbólico pode ser a formação de classes.

Ao descobrir que relações arbitrárias ensinadas davam origem, sem ensino explícito, a novas relações arbitrárias coerentes com as primeiras, Sidman e seus colaboradores (Sidman, 1971; Sidman \& Tailby, 1982; Sidman, Wilson-Morris $\&$ Kirk, 1986) começaram a pesquisar como e por que o ensino das relações originais produzia as relações derivadas. Foi como abrir uma Caixa de Pandora. O estudo comportamental desse tipo de relações de equivalência tornou-se um modelo experimental para estudos sobre comportamento simbólico, linguagem e cognição.

Ao longo da história da nossa espécie, desenvolvemos a nossa capacidade de abstração paralelamente ao desenvolvimento da linguagem, a capacidade de responder a um estímulo "como se" fosse outro, isto é, a substituição ou equivalência de estímulos, assumiu uma grande importância, fundamental para o desenvolvimento cognitivo.

Apesar disso, os procedimentos experimentais não têm sido adequados para acessar o desempenho de usar a equivalência em não-humanos, o que permitiria analisar com mais precisão esse repertório especialmente importante para a espécie humana. Algumas poucas demonstrações convincentes de equivalência com organismos não-humanos foram obtidas com um leão marinho (Kastak, Schusterman \& Kastak, 2001; Schusterman \& Kastak, 1993) mas não foram ainda replicadas com animais da mesma ou de outra espécie. Ainda há dúvida quanto aos aspectos do procedimento de ensino responsáveis pela obtenção do repertório.

O programa de pesquisa "Escola Experimental de Primatas" tem como objetivo desenvolver uma seqüência de procedimentos de ensino através da qual seja possível construir, de maneira replicável, repertórios complexos como "usar equivalências" em indivíduos não-humanos. O desenvolvimento destes procedimentos de ensino pode contribuir para conhecer melhor como desenvolver repertórios complexos em pessoas com desenvolvimento atrasado. 
No "curriculum" da Escola Experimental de Primatas, o objetivo da etapa de treino de relações condicionais arbitrárias será o de levar os participantes a lidar com uma rede de relações arbitrárias entre estímulos, demonstrando a capacidade de substituir um estímulo por outro, colocando-os na mesma classe apenas com base em uma história de relações condicionais arbitrárias inter-relacionadas, reproduzindo, com primatas como aprendizes, a complexa rede de relações de equivalência e compreender como ela se desenvolve.

A analogia do laboratório com a escola tem o objetivo de chamar a atenção para as similaridades entre as atividades desenvolvidas no programa de pesquisa e as atividades que são (ou deveriam ser) comuns numa escola convencional: os procedimentos de ensino e os resultados são objeto de avaliação ao longo do processo de ensino, gerando aperfeiçoamentos e correções; os procedimentos são planejados para ensinar uma seqüência hierárquica de repertórios em que cada novo desempenho aprendido é pré-requisito para os seguintes.

Os procedimentos de ensino pretendem ser modelos simplificados das condições para a aprendizagem de relações condicionais com humanos normais (adultos e crianças) e pessoas com problemas de aprendizagem, atraso escolar e deficiência mental. Partimos do suposto que estaremos aumentando a nossa compreensão sobre como organizar as condições de ensino, sem depender de instruções verbais, para indivíduos com dificuldades de aprendizagem em geral, se formos capazes de levar macacos-prego a exibir repertórios que sejam pré-requisitos para repertórios complexos.

Seqüencialmente, ensinam-se discriminações simples, discriminações condicionais de identidade, discriminações condicionais arbitrárias isoladas e discriminações condicionais arbitrárias inter-relacionadas, como será detalhado abaixo, tomando os cuidados metodológicos para garantir que o controle efetivo do desempenho seja o planejado.

Analisando como o desempenho evolui em função das condições experimentais programadas, reuniremos informações sobre os processos comportamentais envolvidos no desenvolvimento do funcionamento cognitivo normal, difíceis de obter fora do laboratório.

\section{Método}

\section{Participantes}

No biotério de primatas do Laboratório de Psicologia da UFPA são mantidos, em quatro gaiolas-viveiro, nove macacos da espécie Cebus apella, oito machos e uma fêmea, provindos do Centro Nacional de Primatas.

A classificação desses animais em sub-espécies é uma questão controversa (Rylands, Schneider, Langguth, Mittermeier, Groves \& Rodrigues-Luna, 2000). Em seu habitat "o tamanho do grupo no gênero $C$. apella varia de indivíduos solitários até 50 ou mais" (Lynch \& Rímoli, 2000, p.45). Estes animais vivem em grupos com um macho e uma fêmea dominantes, em uma grande variedade de habitats; exploram tanto a copa das árvores como o chão na sua rotina diária; possuem presas poderosas, eficientes para caça, abertura de crustáceos e castanhas, bem como em interações agonísticas entre indivíduos da mesma espécie. Com alimentação preferencialmente baseada em frutos, adaptam-se com facilidade aos alimentos disponíveis, incluindo pequenos animais, raízes, flores e brotos (Rímoli, 2001). Do ponto de vista comportamental, os macacos-prego possuem uma relativa facilidade em desempenhar tarefas, tanto em seu ambiente natural, como em laboratório (Freese \& Oppenheimer, 1981), mas discute-se se sua capacidade cognitiva não ficaria restrita a aprendizagens de caráter associativo, não generalizáveis (Visalberghi \& Tomasello, 1998).

\section{Condições de manutenção}

Os participantes: Juninho (M06) e Ari ET (M07), ambos adultos, moram em uma gaiola-viveiro; Guga (M09) e Raul (M14), jovens, vivem em uma outra gaiola; Cotó (M12) e Drácula (M13), também jovens, dividem uma terceira gaiolaviveiro; Louis (M15) e Bongo (M16) vivem na quarta gaiola, na qual vive também uma fêmea jovem, Baguette, que ainda não participou dos experimentos.

As gaiolas-viveiro, construídas em tela metálica sobre uma base de alvenaria, ficam na área externa à sala de coleta de dados, têm metade de sua área coberta com telhas de fibrocimento. As três primeiras gaiolas-viveiro medem 2,57 x 1,83 x $1,85 \mathrm{~m}$. A quarta mede 4,00 x 1,83 x 1,85 m. No interior de cada uma delas, ao longo das grades laterais e do fundo, e a $1 \mathrm{~m}$ de altura do solo, há plataformas de ripas de madeira. Sobre uma das plataformas, na parte coberta, fica um abrigo de madeira de 0,47 x 0,49 x 0,47 m, aberto em um dos lados. Cada gaiolaviveiro tem um suporte para bandeja medindo $0,24 \times 0,33 \mathrm{x}$ $0,24 \mathrm{~m}$, um bebedouro de bico de aço e, anexa à parede lateral, a $1 \mathrm{~m}$ de altura do solo, uma gaiola de transporte medindo 1,20 x 0,80 x $0,55 \mathrm{~m}$, com portas de correr dando acesso para o interior da gaiola-viveiro e para o exterior, utilizada para abrigar os macacos quando da limpeza da gaiola-viveiro ou quando serão conduzidos às sessões experimentais.

A dieta diária fornecida uma vez ao dia aos participantes é composta de frutas e legumes, cortados em pedaços, enriquecida em dias alternados com ovos cozidos, castanhado-pará, ração canina com $28 \%$ de proteínas, leite integral e, semanalmente, uma dose de complexo vitamínico adicionado à água, que é fornecida ad libitum. Três horas após o alimento ter sido fornecido, os resíduos são retirados da gaiolaviveiro, por razões de higiene e saúde, e para assegurar que na hora da realização de sessões experimentais, os animais estejam privados de alimento. Acadêmicos de medicina veterinária mantêm vigilância sanitária e providenciam exames rotineiros e especiais, diagnóstico e medicação quando necessária, sob supervisão.

\section{Ambiente experimental}

As sessões para a coleta de dados são realizadas em duas câmaras experimentais, cada uma medindo 0,80 x 0,80 x $0,70 \mathrm{~m}$. Um monitor de tela sensível ao toque fica acoplado, pelo lado externo, a uma janela na câmara experimental, repousando sobre uma prateleira. No canto superior direito da parede oposta ao monitor de vídeo, fica uma lâmpada fluorescente de 15 watts e, no canto superior esquerdo, um suporte 
Tabela 1.

Participantes e estágio de ensino atingido até 12/2001

\begin{tabular}{|c|c|c|c|}
\hline $\begin{array}{c}\mathrm{N}^{\circ} \text { de } \\
\text { Matrícula }\end{array}$ & Nome & $\begin{array}{l}\text { Idade em meses } \\
\text { em } 12 / 2000\end{array}$ & Estágio Atual \\
\hline M06 & Juninho & 76 & Identidade Generalizada \\
\hline M07 & ET & 76 & Identidade Generalizada \\
\hline M09 & Guga & 18 & Identidade Generalizada \\
\hline M12 & Cotó & 18 & Identidade Generalizada \\
\hline M13 & Drácula & 18 & $\begin{array}{l}\text { Reversão de Discriminação Simples } \\
\text { com } 2 \text { Estimulos }\end{array}$ \\
\hline M14 & Raul & 18 & Identidade Generalizada \\
\hline M15 & Louis & & $\begin{array}{l}\text { Identidade Generalizada* e } \\
\text { Simetria }\end{array}$ \\
\hline M16 & Bongo & & $\begin{array}{l}\text { Reversão de Discriminação Simples } \\
\text { com } 2 \text { Estímulos }\end{array}$ \\
\hline
\end{tabular}

metálico para uma câmara filmadora. Essa lâmpada, e duas lâmpadas fluorescentes de 40 watts no teto da sala acima da câmara experimental, ficam acesas durante as sessões. $\mathrm{Na}$ parede lateral esquerda, há uma porta de $0,35 \times 0,20 \mathrm{~m}$, utilizada como entrada e saída do sujeito da câmara experimental.

Ligado ao monitor, há um computador 486 DX2 66, com um aplicativo intitulado TREL versão 2.1 (criado por José Iran A. dos Santos), especificamente desenvolvido para experimentos envolvendo treino de relações entre estímulos, com tentativas de escolha entre até quatro estímulos, o qual é utilizado para a apresentação dos estímulos e registro das respostas e latências. As escolhas corretas são "recompensadas" com pelotas de $190 \mathrm{mg}$ com sabor e aroma de banana (Noyes food pellets) através de um dispensador automático cujo depósito de pelotas e mecanismo ficam do lado de fora da câmara experimental. Através de uma mangueira, a pelota chega até uma bandeja situada $24 \mathrm{~cm}$ abaixo do monitor de vídeo, dentro da câmara experimental. Logo acima da bandeja do comedouro há uma luz vermelha que acende no momento em que o comedouro é acionado.

As duas câmaras experimentais têm pequenas diferenças entre elas. Por isso, os mesmos animais freqüentam sessões sempre na mesma câmara experimental.

\section{Procedimento: a seqüência de ensino}

\section{Adaptação à rotina da pesquisa}

Ao chegarem à Escola, os macacos permanecem em observação e sob cuidados médico-veterinários de rotina e se adaptam às condições de alojamento, alimentação e vida em grupo. O alojamento em pares tem se mostrado vantajoso, minimizando as brigas por dominância e a dificuldade de acesso aos alimentos.

As pessoas que têm acesso às gaiolas receberam instruções estritas de não fazer gestos bruscos, mesmo em casos de fuga de animais, e de não agredi-los. Toda manipulação é feita com o uso de iscas alimentares. Dessa maneira, os ani- mais desenvolveram comportamentos interativos com os experimentadores, exibindo baixa agressividade. Um estudo do comportamento social desses macacos-prego em cativeiro está em andamento.

\section{Treino de Manejo}

O primeiro procedimento de ensino é o de manejo, envolvendo a passagem da gaiola-viveiro para a gaiola de transporte, desta para a de transporte e vice-versa. Respostas cada vez mais próximas das respostas desejadas são recompensadas com alimento. O procedimento consiste no reforçamento diferencial por aproximações sucessivas de uma resposta final (Skinner, 1989).

\section{Treino ao comedouro e modelagem da resposta de tocar os estímulos}

Na câmara experimental, cada macaco aprende a encontrar, pegar e comer a pelota de ração que cai na bandeja mediante acionamento do dispensador, quando ouve o ruído da liberação da pelota (treino ao comedouro).

Em seguida ensinamos o animal a tocar um quadrado branco de $4 \times 4 \mathrm{~cm}$, projetado sobre um fundo preto na tela do monitor, usando o procedimento de modelagem, como no treino de manejo. O dispensador de pelotas é acionado cada vez que o animal apresenta movimentos cada vez mais próximos do desejado, que é tocar a tela na área do quadrado branco.

Tão logo a resposta de tocar o estímulo comece a ocorrer, ela é sempre recompensada. Até esse momento, o que se requer dos macacos é tão somente que eles atentem para a presença de algo na tela do computador e respondam a esse estímulo.

\section{Discriminações simples com pares e trincas de estímulos}

Uma vez assegurado o repertório de vir às sessões experimentais, e tocar nos estímulos apresentados na tela do monitor, pegar e comer a pelota de ração dispensada na bandeja, e esperar a apresentação do próximo estímulo, o ambi- 
ente está preparado para começarmos a apresentar problemas de escolha entre estímulos.

Como o macaco trabalha para obter suas pelotas, e tende a repetir as respostas que as produzem, o arranjo experimental nos permite "informar" aos nossos animais se a escolha que ele efetuou foi correta, simplesmente usando o expediente de acionar o dispensador como conseqüência para as respostas de tocar o estímulo que definimos como correto, e não fazê-lo se a resposta de escolha foi em direção a um outro estímulo. Ao aprender a tocar o quadrado branco e não o restante da tela o animal está se preparando para aprender situações de escolha mais difíceis, entre dois quadrados em cores diferentes, por exemplo.

O primeiro problema de escolha envolve a apresentação simultânea de dois estímulos diferentes na tela, sendo que tocar em um deles aciona o dispensador e uma pelota cai na bandeja e pode ser consumida. Após um pequeno intervalo os mesmos estímulos são apresentados de novo, em locais diferentes da tela.

Resolvido o primeiro problema nós o invertíamos e o estímulo que era correto anteriormente passava a ser o incorreto e o estímulo definido como incorreto passava a ser correto. Apresentava-se ao participante tantas tentativas quantas fossem necessárias para ele aprender a reversão da discriminação anterior, isto é, a escolher consistentemente o estímulo que anteriormente ele havia aprendido a preterir e que agora era o correto.

O procedimento de reversão da discriminação era repetido várias vezes com o mesmo par, e depois com novos pares, e depois com trincas de estímulos, com um estímulo correto e dois incorretos.

O objetivo dessa fase era de que os macacos aprendessem a 1) atentar para o conteúdo das figuras-estímulo e 2) reverter as discriminações, ou seja, reverter rapidamente o estímulo escolhido quando o estímulo que era correto passa a ser errado e vice-versa. Essa era uma importante habilidade necessária para resolver os próximos problemas.

\section{Discriminações condicionais por identidade}

Depois de estarem resolvendo os problemas de discriminações simples entre dois e entre três estímulos simultaneamente, com mudanças repetidas no estímulo correto, passamos a apresentar, com os mesmos estímulos, tentativas em duas etapas. Na primeira etapa, era apresentado um estímulo sozinho, como modelo, e o participante tinha que tocar esse estímulo. Então ele desaparecia e os estímulos de comparação eram apresentados, dentre os quais um igual ao modelo. Se o animal tocasse o estímulo de comparação igual ao modelo, uma pelota de comida era apresentada. Após um pequeno intervalo, um novo estímulo modelo era apresentado e novamente a resposta correta era a de escolher o estímulo de comparação igual ao modelo. Esse é um procedimento conhecido como "procedimento de escolha de acordo com o modelo" ou "emparelhamento ao modelo" (Carter \& Werner, 1978; Cumming \& Berryman, 1965).

Além de ser capaz de diferenciar um estímulo do outro, o participante tinha agora que escolher um estímulo diferente a cada tentativa; o estímulo correto era condicional ao modelo. Por isso dizemos que ao resolver esse tipo de problema, o macaco está demonstrando discriminações condicionais (Lashley, 1938), pois se um determinado estímulo é correto ou não depende de uma condição estabelecida pela apresentação do estímulo modelo.

O objetivo desta fase é que animais aprendam a responder à igualdade entre os estímulos, ou seja, a selecionar, dentre os estímulos de comparação, aquele idêntico ao modelo.

Os sujeitos aprendem as discriminações condicionais de identidade, e o efeito do grau de semelhança entre os estímulos sobre a precisão do desempenho aparece, com a aprendizagem mais rápida com estímulos que guardam maior diferença entre si.

De qualquer maneira, para responder à pergunta levantada, se os nossos animais aprendem a escolher cada par de estímulos ou aprendem o conceito de identidade, é necessário apresentar os problemas de escolha por identidade utilizando estímulos novos, desconhecidos para os participantes, ou seja, é necessário efetuar testes de identidade generalizada e isso foi feito.

\section{Testes de identidade generalizada}

Após atingir o critério de aprendizagem na fase anterior, de dezoito tentativas corretas consecutivas, estímulos novos eram apresentados nas mesmas condições de escolha de acordo com o modelo. Cada escolha correta era recompensada. Inicialmente usamos estímulos que já haviam sido apresentados antes nas discriminações simples, mas que nunca haviam sido apresentados na situação de escolha por identidade com o modelo e, posteriormente, estímulos completamente novos. Se o macaco exibia prontamente o desempenho de escolha por identidade com os estímulos novos, podíamos dizer que eles apresentavam o desempenho de escolha por identidade generalizada.

\section{Relações condicionais arbitrárias}

Nesta etapa, demos um passo a mais no sentido de verificar a possibilidade de ensinar os pré-requisitos da abstração aos animais. Usamos um modelo de discriminação condicional no qual não é mais a identidade entre o modelo e uma das comparações que define qual das comparações é a correta. Usamos, inicialmente, dois conjuntos de três estímulos, sendo que os estímulos de um dos conjuntos funcionavam como estímulos modelo e os estímulos do outro conjunto funcionavam como estímulos de comparação. Não havia, portanto, uma relação de semelhança física entre os estímulos modelo e de comparação correspondentes; essa relação era arbitrariamente definida pelos experimentadores.

Esse tipo de relação pretende replicar experimentalmente as características arbitrárias das relações entre nomes de objetos, de pessoas ou de eventos e os objetos, pessoas ou eventos propriamente ditos, ou mesmo das relações entre as letras e os sons na linguagem escrita.

\section{Testes de Simetria}

Demonstrar que nossos animais eram capazes de demonstrar identidade generalizada e de aprender relações arbitrárias 
foram os primeiros passos para demonstrar as características da formação de classes de equivalência.

Depois de aprender um conjunto de relações modelocomparação, já é possível testar uma relação não explicitamente ensinada, mas que pode ter emergido como resultado do ensino do primeiro conjunto de relações condicionais arbitrárias e como indicação de que os estímulos relacionados condicionalmente passaram a ser substituíveis um pelo outro: tratase da relação inversa, ou seja, simétrica, da relação aprendida.

Para o resultado ser positivo, em primeiro lugar os animais devem ter aprendido a relação condicional ensinada como uma relação condicional mesmo, fazendo suas escolhas conforme o modelo, e não memorizando pares de estímulos. Em segundo lugar, é necessário que o controle contextual no momento do teste leve o animal a escolher conforme o modelo sob influência da aprendizagem anterior, e não como se fosse uma nova situação, com novas relações a aprender. Estará o aprendiz preparado para entender a pergunta implícita nesses testes de simetria, dando-nos indícios de emergência de comportamento simbólico?

\section{Testes de Transitividade e Simetria da Transitividade}

Uma vez que ensinamos, em nossa "Escola", as primeiras relações condicionais arbitrárias, os primeiros testes de substituição dos estímulos (simetria, neste caso) puderam ser efetuados, para verificar se o ensino das relações condicionais entre os estímulos havia sido suficiente para que, também, a relação simétrica passasse a fazer parte do repertório do participante.

Para verificar se a relação transitiva surge, entretanto, precisaremos - isto está nos nossos planos - ensinar pelo menos mais um conjunto de relações condicionais, tomando os estímulos que haviam sido usados como comparação na fase anterior de ensino, e usando-os como estímulos modelo, relacionando-os a novos estímulos de comparação. Dessa forma poderemos ver se os dois grupos de estímulos - os que foram modelo no primeiro conjunto e os que foram comparação no segundo conjunto de relações ensinadas - que o participante aprendeu a relacionar condicionalmente ao mesmo grupo de estímulos também se relacionam condicionalmente, sem que essa relação seja ensinada diretamente.

$\mathrm{O}$ teste da propriedade transitiva, como vimos, requer dois conjuntos de relações condicionais entre três conjuntos de estímulos, e admite dois níveis de dificuldade: a transitividade direta, em que se verifica se emerge a relação condicional entre os estímulos que eram modelo na primeira fase e os estímulos que eram comparação na segunda fase de ensino, e a simetria da transitividade, em que os estímulos de comparação da segunda fase de ensino servem, no teste, como modelo, e os estímulos que haviam sido modelo na primeira fase de ensino servem como comparação.

\section{Resultados}

Apesar de terem sido adotados procedimentos sistemáticos para o treino das respostas instrumentais previstas no treino de manejo, treino ao bebedouro e modelagem da resposta de tocar a tela do computador, nossos procedimentos de registro eram mais rigorosos a partir do momento em que os primeiros "problemas" de discriminação eram apresentados (treino de discriminações simples simultâneas com pares de estímulos). O propósito do presente texto não é apresentar e analisar os dados experimentais produzidos na Escola Experimental de Primatas, ao longo destes anos; é, na verdade, apresentar didaticamente uma estratégia incomum de lidar com a pesquisa sobre comportamento complexo com animais (uma abordagem educacional, ver Barros, Galvão \& McIlvane, no prelo) e destacar as possibilidades de aplicação do conhecimento extraído destes estudos.

Assim, nesta sessão de resultados e discussão, complementamos os comentários que já vêm sendo feitos sobre os avanços no comportamento dos nossos sujeitos e apresentamos alguns dados relativos a testes de identidade generalizada, como ume espécie de amostra (para obter dados adicionais deste programa de pesquisa ver, por exemplo, Barros, 1998 e Barros, Galvão \& McIlvane, 2002).

A respeito do treino de discriminações simples, de maneira geral, cada novo problema de escolha era eventualmente resolvido pelos macacos depois de um certo número de tentativas. A dificuldade era proporcional à similaridade entre os estímulos. Quanto maior a diferença entre os estímulos, mais rápida a aprendizagem.

Seis sujeitos, M06, M07, M09, M12, M14 e M15, mostraram desempenho de escolha da comparação idêntica ao modelo com estímulos novos. Quatro deles o fizeram em condições em que todas as escolhas eram reforçadas, e um deles, M15 - Louis, demonstrou o desempenho de escolha das comparações idênticas ao modelo com estímulos novos, sem reforçamento para as escolhas. Esses mesmos e os outros sujeitos deverão passar por uma nova etapa de ensino em que parte das escolhas corretas não é reforçada e as tentativas de teste, com estímulos novos, substituem algumas das tentativas com estímulos conhecidos.

Na Tabela 2 apresentamos, para cada sujeito, o número de respostas a cada comparação, por modelo, nas tentativas de linha de base e de teste, em uma sessão de teste de identidade generalizada para cada sujeito. Todos os sujeitos passaram por diversas sessões de teste, cujos resultados podem ser vistos em Barros e colaboradores (2002). Os resultados mostrados aqui, de uma sessão de teste por sujeito, mostram detalhes do controle de estímulos nas sessões de teste, apontando o número de vezes em que cada comparação foi escolhida nas tentativas da linha de base e de teste.

\section{Discussão}

A presente abordagem comportamental ao estudo da cognição é compatível com definições geralmente aceitas de cognição, e os resultados até aqui obtidos mostraram que, dadas as condições adequadas, macacos prego são capazes de adquirir o conceito de igualdade (Barros et al., 2002), operacionalizado no desempenho de identidade generalizada, assim como já foi possível demonstrar desempenhos de 
Tabela 2 .

Número de respostas a cada comparação, por modelo, nas tentativas de linha de base e de teste, em uma sessão de teste de identidade generalizada de cada sujeito.

\begin{tabular}{|c|c|c|c|c|c|c|c|c|}
\hline \multirow[b]{3}{*}{ Sujeito } & \multirow[b]{3}{*}{ Modelo } & \multicolumn{3}{|c|}{ Linha de Base } & \multicolumn{4}{|c|}{ Teste } \\
\hline & & \multicolumn{3}{|c|}{ Comparações } & \multirow[b]{2}{*}{ Modelo } & \multicolumn{3}{|c|}{ Comparações } \\
\hline & & $\mathrm{Al}$ & $\mathrm{A} 2$ & $\mathrm{~A} 3$ & & $\mathrm{~B} 1$ & $\mathrm{~B} 2$ & B3 \\
\hline \multirow[t]{4}{*}{ M06 } & Al & 12 & - & - & B1 & 9 & 2 & 1 \\
\hline & A2 & - & 12 & - & B2 & - & 9 & 3 \\
\hline & A3 & 2 & . & 10 & B3 & 1 & 1 & 10 \\
\hline & & $\mathrm{Al}$ & $\mathrm{A} 2$ & $\mathrm{~A} 3$ & & $\mathrm{~B} 1$ & $\mathrm{~B} 2$ & B3 \\
\hline \multirow[t]{3}{*}{ M07 } & $\mathrm{A} 1$ & 12 & - & - & BI & 11 & & 1 \\
\hline & $\Lambda 2$ & - & 11 & 1 & B2 & 2 & 8 & 2 \\
\hline & A3 & 2 & - & 10 & B3 & 3 & 1 & 8 \\
\hline \multirow[t]{4}{*}{ M09 } & & $\mathrm{Cl}$ & $\mathrm{C} 2$ & $\mathrm{C} 3$ & & D1 & $\mathrm{D} 2$ & D3 \\
\hline & $\mathrm{Cl}$ & 12 & - & - & D1 & 10 & 1 & 1 \\
\hline & $\mathrm{C} 2$ & - & 12 & - & D2 & - & 12 & - \\
\hline & $\mathrm{C} 3$ & - & - & 12 & D3 & 1 & 1 & 10 \\
\hline \multirow[t]{4}{*}{ M12 } & & $\mathrm{Al}$ & $\mathrm{A} 2$ & A3 & & $\mathrm{Cl}$ & $\mathrm{C} 2$ & $\mathrm{C} 3$ \\
\hline & Al & 12 & - & - & $\mathrm{Cl}$ & 11 & - & 1 \\
\hline & $\mathrm{A} 2$ & - & 12 & - & $\mathrm{C} 2$ & 1 & 10 & 1 \\
\hline & A3 & - & - & 12 & $\mathrm{C} 3$ & 4 & - & 8 \\
\hline \multirow[t]{4}{*}{ M14 } & & $\mathrm{A} 1$ & $\mathrm{~A} 2$ & A3 & & E1 & E2 & E3 \\
\hline & A1 & 8 & - & - & E1 & 5 & 1 & 2 \\
\hline & A2 & - & 9 & - & F2 & 1 & 7 & - \\
\hline & A3 & - & - & 7 & E3 & - & - & 8 \\
\hline \multirow[t]{4}{*}{ M15 } & & $\mathrm{Fl}$ & $\mathrm{F} 2$ & F3 & & G1 & $\mathrm{G} 2$ & G3 \\
\hline & $\mathrm{F} 1$ & 18 & 2 & - & $\mathrm{G} 1$ & 4 & - & - \\
\hline & F2 & 1 & 17 & 2 & G2 & - & 3 & 1 \\
\hline & F3 & - & - & 20 & $\mathrm{G} 3$ & 1 & - & 3 \\
\hline
\end{tabular}

escolha por identidade generalizada em chimpanzés (Oden, Thompson \& Premack, 1988) e leões-marinhos (Kastak \& Schusterman, 1994).

De acordo com Barros e colaboradores (2002), a análise dos relatos de insucessos em produzir pareamento ao modelo por identidade generalizado em animais aponta os seguintes problemas experimentais a serem resolvidos:

1. Ao invés de acertar por emparelhar a comparação ao modelo, o sujeito aprende a escolher a comparação correta em cada configuração de estímulos. Dessa forma, a posição passa a fazer parte dos estímulos. Esse desempenho não resiste a mudanças, por exemplo, na localização dos estímulos. Para resolver esse problema, variamos as posições dos estímulos, inclusive do estímulo modelo, tornando virtualmente impossível a aprendizagem de "configurações".

2. As escolhas durante o ensino da discriminação ou nos testes com estímulos novos sofrem a influência da similaridade entre os estímulos. Um efeito de generalização ocorre. Para contornar essa fonte de controle das escolhas, os estímulos de cada conjunto são cuidadosamente escolhidos, e quando a discriminação entre dois estímulos em particular se torna demorada, um dos estímulos é trocado.

3. Diferença pronunciada entre o contexto de ensino e manutenção e o contexto de teste, gerando um "efeito de novidade", que gera respostas que interferem com as respostas de escolha. Para sanar esse problema, as sessões que antecedem o teste são o mais similares possível às de teste. Por exemplo, os testes com estímulos novos são feitos com a mesma probabilidade de reforço da linha de base. Antes de um conjunto de estímulos ser apresentado na situação de pareamento ao modelo, o sujeito aprende a escolher esses estímulos em situações de discriminação simples, de forma que a novidade no teste se restringe ao procedimento de discriminação condicional.

A demonstração até aqui alcançada, de identidade generalizada, nos anima a supor que estamos no caminho correto 
para decifrar as condições necessárias para detectar a formação de classes de estímulos com animais e, assim, eliminar a hipótese de que a emergência de relações arbitrárias entre estímulos, uma das bases das operações com símbolos, dependa de nomeação e linguagem (Carr, Wilkinson, Blackman \& McIlvane, 2000; Sidman, 2000).

A identificação das condições necessárias para a construção desse tipo de repertório com primatas não-humanos, na medida em que envolve o desenvolvimento de tecnologia comportamental para desenvolver controle contextual sem a necessidade de instruções verbais, poderá ser útil na determinação das condições necessárias para a obtenção de repertório simbólico com pessoas com problemas de desenvolvimento lingüístico (Carr et al., 2000).

A teoria da coerência de controle de topografia de controle de estímulos (McIlvane, Serna, Dube \& Stromer, 2000), explicita as condições para garantir que o controle de estímulos planejado pelo experimentador coincida com o controle de estímulos desenvolvido pelo sujeito. O planejamento de ensino passo a passo, levando em consideração o desempenho em cada etapa precedente, já se mostrou eficiente para a obtenção da emergência de desempenho de identidade generalizada. Agora estamos seguindo para o ensino de relações arbitrárias, com a mesma abordagem geral.

Ainda é cedo para aceitar que o macaco prego não seja capaz de fazer relações abstratas entre estímulos. Se é certo que esses indivíduos jamais atingirão o nível de desempenho intelectual humano, definir a priori os limites de suas possibilidades com base em demonstrações de insucessos é desprezar a tecnologia de ensino e as possibilidades hoje existentes de estender o repertório de animais, ou de indivíduos com retardo mental, muito além do que se imaginou possível há pouco tempo atrás.

\section{Agradecimentos}

O programa de pesquisa relatado neste artigo foi apoiado pela UFPA, com bolsas de IC para o último autor, CNPq, através de Bolsas de Produtividade em Pesquisa para o primeiro e segundo autores, e bolsas de IC para o terceiro e quarto autores, e pelo MCT/PRONEX 2, pelo financiamento do projeto "Relações emergentes entre estímulos e suas aplicações ao ensino de leitura, escrita e matemática". Agradecemos a Jorgete Lopes da Silva, Sandra Brandão, Cíntia Lavratti, José Ricardo pela colaboração na coleta de dados e participação nas discussões, a Cibele Colino Magalhães pela assistência veterinária e Edilson Ferreira Pastana pelo cuidado com os animais.

\section{Referências}

Barros, R. S. (1998). Controle do comportamento por relações entre estímulos em Cebus apella. Tese de Doutorado. São Paulo: Universidade de São Paulo.<www.teses.usp.br>

Barros, R. S., Galvão, O. F., \& McIlvane, W. V. (2002). Generalized identity matching-to-sample in Cebus apella. The Psychological Record, 52, 441-460.

Barros, R. S., Galvão, O. F., \& McIlvane, W. J. (no prelo). The search for relational learning capacity in Cebus apella: A programmed "educational" approach. In Soraci, S. A. (Org.), Perspectives on fundamental processes in intellectual functioning, Vol. II. Stanford: Ablex.

Bates, E. (1979). The emergence of symbols: Cognition and communication in infancy. New York: Academic Press.

Bussab, V. S. R. (2000). Fatores hereditários e ambientais no desenvolvimento: A adoção de uma perspectiva interacionista. Psicologia: Reflexão e Crítica, $13,233-243$

Bussab, V. S. R. \& Ribeiro, F. L. (1998). Biologicamente cultural. In L. Souza, M. F. Q. Freitas \& M. M. P. Rodrigues (Orgs.), Psicologia: reflexões (im)pertinentes (pp.175-193). São Paulo: Casa do Psicólogo.

Carr, D., Wilkinson, K. M., Blackman, D., \& Mcllvane, W. J. (2000). Equivalence classes in individuals with minimal verbal repertoires. Journal of the Experimental Analysis of Behavior, 74, 101-114.

Carter, D. E., \& Werner, T. J. (1978). Complex learning and information processing by pigeons: a critical analysis. Journal of the Experimental Analysis of Behavior, 29, 565-601.

Catania, C. A. (1999). Aprendizagem: comportamento, linguagem e cognição. Porto Alegre: ArtMed.

Chaves, A. M. (2000). O fenômeno psicológico como objeto de estudo transdisciplinar. Psicologia: Reflexão e Crítica, 13, 159-165.

Codo, W. \& Vasques-Menezes, I. (1999). O que é burnout? In W. Codo (Org.), Educação: carinho e trabalho (pp. 237-254). Petrópolis: Vozes.

Cumming, W. W., \& Berryman, R. (1965). The complex discriminated operant: studies of matching-to-sample and related problems. In D. J. Mostofsky (Org.), Stimulus generalization (pp. 284-330). Standford, CA: Standford University Press.

Donahoe, J. W., \& Palmer, D. C. (1994). Learning and complex behavior. Boston: Allyn and Bacon.

Dube, W. V. (1996). Teaching discrimination skills to persons with mental retardation. Temas em Educação Especial, 3, 73-96.

Freese, C. H., \& Oppenheimer, J. R. (1981). The capuchin monkeys, genus Cebus. In A. F. Coimbra-Filho \& R. A. Mittermeier (Orgs.). Ecology and behavior of neotropical primates, Vol I (pp. 331-390). Rio de Janeiro: Academia Brasileira de Ciências.

Jennings, H. S. (1906/1976). Behavior of the lower organisms. Bloomington: Indiana University Press.

Kastak, D., \& Schusterman, R. (1994). Transfer of visual identity matching-tosample in two California sea lions (Zalophus californianus). Animal Learning e Behavior, 22, 427-435.

Kastak, C. R., Schusterman, R. J., \& Kastak, D. (2001). Equivalence classification by California sea lions using class-specific reinforcers. Journal of the Experimental Analysis of Behavior, 76, 131-158.

Lashley, K. S. (1938). Conditional reactions in the rat. Journal of Psychology, 6 , 311-324.

Lionello, K. M.. \& Urcuioli, P. J. (1998). Control by sample location in pigeons' matching to sample. Journal of the Experimental Analysis of Behavior, 70, 235-251.

Lynch, J. W., \& Rímoli, J. (2000). Demography of a group of tufted capuchin monkeys (Cebus apella nigritus) at the Estação Biológica de Caratinga, Minas Gerais, Brazil. Neotropical Primates, 8, 44-49.

McIlvane, W. J., \& Cataldo, M. F. (1996). On the clinical relevance of animal models for the study of human mental retardation. Mental Retardation and Developmental Disabilities Research Reviews, 2, 188-196.

McIlvane, W. J., Serna, R. W., Dube, W. V., \& Stromer, R. (2000). Stimulus control topography coherence and stimulus equivalence: Reconciling test outcomes with theory. In J. Leslie e D. E. Blackman (Orgs.), Issues in experimental and applied analysis of human behavior (pp. 85-110). Reno: Context.

Oden, D. L., Thompson, R. K. R., \& Premack, D. (1988) Spontaneous transfer of matching by infant chimpanzees (Pan troglodytes). Journal of Experimental Psychology: Animal Behavior Processes, 14, 140-145.

Peeke, H. V. S., \& Herz, M. J. (Orgs.) (1973). Habituation: physiological substrates, Vol. 2. New York: Academic Press. 
Rímoli, J. (2001). Ecologia de macacos-prego (Cebus apella nigritus, Goldfuss, 1809) na Estação Biológica de Caratinga (MG): implicações para a conservação de fragmentos de Mata Atlântica. Tese de Doutorado não-publicada. Universidade Federal do Pará, Belém.

Rylands, A. B., Schneider, H., Langguth, A., Mittermeier, R. A., Groves, C. P., \& Rodriguez-Luna, E. (2000). An assessment of the diversity of New World primates. Neotropical Primates, 8, 61-93.

Schusterman, R. J., \& Kastak, D. (1993). A California sea lion (Zalophus californianus) is capable of forming equivalence relations. The Psychological Record, 43, 823-839.

Serna, R. W., Dube, W. V., \& McIlvane, W. J. (1997). Assessing same/differen judgements in individuals with severe intelectual disabilities: A status report. Research in Developmental Disabilities, 18, 343-368.

Sidman, M. (1971) Reading and auditory-visual equivalence. Journal of Speech and Hearing Research, 14, 5-13.

Sidman, M. (1994). Equivalence relations and behavior: A research story. Boston: Authors Cooperative.
Sidman, M. (1995). Coerção. Campinas: Editorial Psy II.

Sidman, M. (2000). Equivalence relations and the reinforcement contingency. Journal of the Experimental Analysis of Behavior, 74, 127-146.

Sidman, M., \& Tailby, W. (1982). Conditional discrimination vs. matching-tosample: an expansion of the testing paradigm. Journal of the Experimental Analysis of Behavior, 37, 5-22.

Sidman, M., Wilson-Morris, M., \& Kirk, B. (1986) Matching-to-sample procedures and the development of equivalence relations: the role of warning. Analysis and Intervention in Developmental Disabilities, 6, 1-20.

Skinner, B. F. (1989). Ciência e comportamento humano. São Paulo: Martins Fontes.

Skinner, B. F. (1991). Sobre o behaviorismo. São Paulo: Cultrix.

Visalberghi, E., \& Limongelli, L. (1994). Lack of compreension of cause-effect relations in tool-using capuchin monkeys (Cebus apella). Journal of Comparative Psychology, 108, 15-22.

Visalberghi, E., \& Tomasello, M. (1998). Primate causal understanding in the physical and psychological domains. Behavioural Processes, 42, 189-203.

Olavo de Faria Galvão, doutor em Psicologia Experimental pela Universidade de São Paulo, é professor do Departamento de Psicologia Experimental da Universidade Federal do Pará, PA.

Romariz da Silva Barros, doutor em Psicologia Experimental pela Universidade de São Paulo, é professor do Departamento de Psicologia Experimental da Universidade Federal do Pará, PA.

Aline Cardoso Rocha, Mariana Barreira Mendonça e Paulo Roney Kilpp Goulart, bolsistas de Iniciação Científica (ALC da UFPA e MBM e PRKG do CNPq), são estudantes do Curso de Psicologia da Universidade Federal do Pará, PA.

Endereço para correspondência: [OFG] Trav. Castelo Branco, 1116/1004, 66063-080, Belém, PA. Fone/Fax: (91)211.1453, e-mail: ofg@cpgp.ufpa.br. 